\title{
Compensation du membre supérieur : des besoins non satisfaits dans les maladies neuromusculaires
}

\author{
Karima Ahnache
}

Une journée consacrée à la perte de fonction motrice du membre supérieur dans les maladies neuromusculaires a été organisée le 14 novembre 2017 par l'AFM-Téléthon, en collaboration avec Filnemus, la filière de santé des maladies neuromusculaires. Cliniciens, industriels, scientifiques et personnes concernées ont pu témoigner de l'importance d'une reconnaissance et d'une prise en charge adaptée dans l'objectif d'une amélioration de la qualité de vie de la personne.

\section{Le contexte}

Les membres supérieurs (épaules, bras, avant-bras et mains) permettent la réalisation de nombreux actes de la vie quotidienne tels que les gestes courants destinés à satisfaire les besoins fondamentaux, physiologiques et relationnels.

Dans les maladies neuromusculaires, la perte de motricité des épaules, des bras ou des mains va compliquer la réalisation de ces gestes quotidiens.
De ce fait, ces difficultés motrices des membres supérieurs vont avoir un impact important sur la préservation de l'autonomie de la personne et sur sa qualité de vie. Plusieurs activités peuvent être impactées: nutrition, soins, hygiène, communication, déplacements, vie relationnelle et sociale, vie professionnelle.

\section{De la manifestation des premiers signes à l'identification des besoins}

Les atteintes du membre supérieur se manifestent de différentes manières, selon la nature et l'importance des déficiences, la compensation n'est donc pas la même. Elle peut être : figée ou évolutive, distale vers proximale, proximale vers distale, d'évolution lente ou rapide, grave et/ou invalidante...

Elle se manifeste par une hypotonie, une dystrophie, des douleurs, une atrophie musculaire, une intolérance à l'effort ou une fatigabilité... Le plus souvent, le déficit part de la racine du membre vers
Karima Ahnache Pôle Aides Techniques Innovation Direction Actions Auprès des Familles AFM-Téléthon, Évry,

France

Contact

kahnache@

afm-telethon.fr

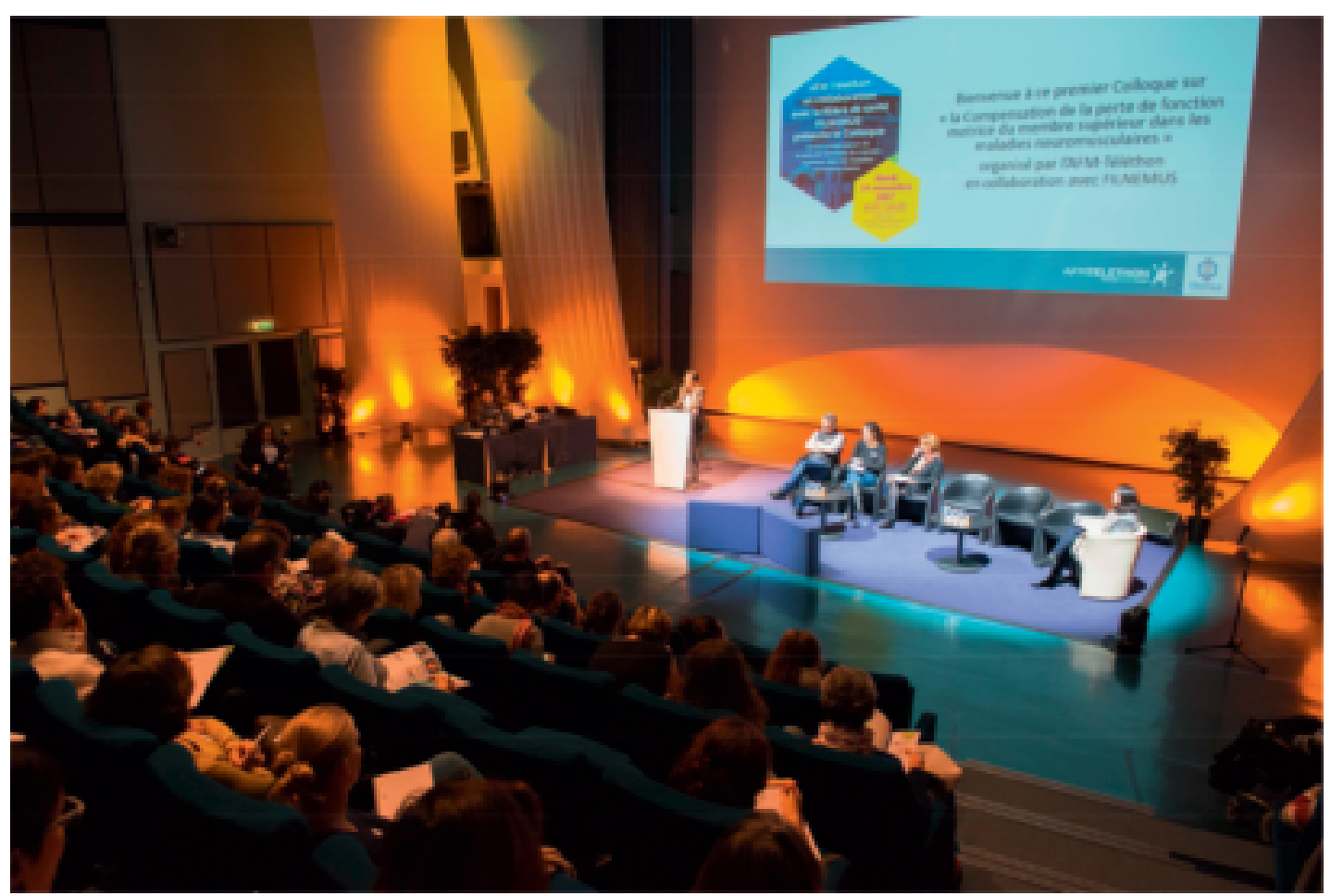


son extrémité. Il ne faut cependant pas négliger le déficit à point de départ distal.

Pour compenser les situations de handicap liées aux déficits du membre supérieur, plusieurs options sont envisageables: la chirurgie et la rééducation, le concours d'une aide humaine, une aide technique. Les situations de handicap peuvent être innées, acquises et/ou acquises par le vieillissement. Ces situations introduisent la notion de technologie de prévention (pour limiter le vieillissement précoce par exemple).

De ces situations de handicap découlent trois sortes de besoins spécifiques au membre supérieur:

- besoin de manipuler les objets (bras embarqués, neuro-prothèses de stimulation),

- besoin d'accéder à l'objet : hauteur, volume, poids (bases mobiles à un ou deux bras),

- besoin d'accéder à l'objet et à l'information (les robots humanoïdes).

\section{Des dispositifs d'assistance multiples et variés}

Une grande diversité d'aides techniques existent et sont disponibles sur le marché pour répondre aux besoins des malades [1].

On en distingue deux grandes catégories :

- les dispositifs de compensation et d'assistance: ce sont des aides techniques qui vont permettre à la personne ayant des capacités motrices résiduelles de pouvoir assister/compléter son mouvement en luttant contre la pesanteur. Les supports de bras mécaniques ou électriques sont conçus pour les personnes atteintes de déficiences musculaires: Top Help $^{\circledR}$, Darwing ${ }^{\oplus}$, Sling ${ }^{\circledR}$ (Focal), Ayura ${ }^{\circledR}$, Edero ${ }^{\circledast}$, Neater Eater $^{\circledast}$ (Ergodiffusion), iFloat ${ }^{\circledR}$ (Domodep) par exemple ;

- les dispositifs de suppléance et de robotique: ce sont des aides techniques qui vont effectuer le mouvement à la place de la personne et suppléer la fonction pour la personne ayant un déficit moteur acquis. Les aides techniques robotisées telles que le Jaco $^{\circledast}$ (Kinova), iArm ${ }^{\circledast}$ (Domodep) ou les aides au repas telles que le Bestic ${ }^{\circledast}$ (Ergodiffusion), le Obi ${ }^{\circledast}$ (Ergodiffusion) vont permettre à la personne de retrouver son autonomie en reproduisant totalement le mouvement à sa place.

\section{Un constat}

Une réflexion collective a été menée au sein de l'AFM-Téléthon entre différents intervenants : Pôle Aides Techniques Innovation, Direction des Actions Médicales, Direction des Affaires Publiques, et Services Régionaux, sur la base d'un constat opéré sur le terrain : bien que les produits existent et soient commercialisés, ils ne sont pas, peu ou mal connus des utilisateurs, des professionnels de terrain, voire même des prescripteurs.

C'est un véritable cercle vicieux qui se dessine avec pour impact: un manque d'information et de connaissance des dispositifs de compensation et de suppléance du membre supérieur qui entraîne une absence de conseils et donc d'évaluations, de préconisation en vue d'obtenir une prescription et, pour finir, une complexité dans l'obtention des financements et donc d'achats de ces dispositifs par les personnes concernées.

\section{Objectifs du colloque}

La réflexion et les travaux menés par l'AFM-Téléthon ces dernières années nous ont amené à proposer l'organisation du colloque "Compensation de la perte de fonctions du membre supérieur dans les maladies neuromusculaires ", dont les objectifs principaux sont la sensibilisation et l'information aux professionnels de santé (médical, paramédical, social) intervenant auprès de nos malades :

- de la nécessité de la bonne prise en charge de ces atteintes fonctionnelles (par une identification des signes précurseurs le plus tôt possible),

- de l'importance de la prise en compte des répercussions fonctionnelles, psychologiques et sociales, - de l'existence de dispositifs d'assistance et de suppléance commercialisés en France afin qu'ils soient prescrits (et remboursés à terme).

La première session a été consacrée au contexte dans lequel s'inscrit ce colloque avec les interventions de médecins et d'ergothérapeutes [2]. La deuxième session a été représentée par les personnes concernées elles-mêmes avec des témoignages, des réalisations, des histoires [3]. Enfin, la troisième session, très internationale, fait l'objet d'une présentation de deux études cliniques sur des dispositifs d'assistance, l'une menée en Italie, l'autre en Hollande, et un projet français de développement d'exosquelette du membre supérieur par les laboratoires d'ingénierie de Saint-Quentin-Versailles.

\section{Une priorité politique et stratégique de l'AFM-Téléthon}

L'Association se soucie plus que jamais de la problématique de la compensation de la perte de fonction motrice des membres supérieurs dans les maladies neuromusculaires. Elle souhaite prioriser ses actions autour de l'accompagnement des personnes dans l'information, le conseil, l'évaluation, la préconisation et enfin la prescription des dispositifs d'assistance non connus ou mal connus des personnes concernées et des professionnels de santé (médecins MPR, ergothérapeutes, kinésithérapeutes, autres). 
Plusieurs objectifs opérationnels sont déjà identifiés : - L'importance pour nos malades de pouvoir accéder à un large panel d'outils à la compensation ou à la suppléance (de l'aide technique la plus simple à l'aide technique la plus sophistiquée tels que les robots mono-tâches aux bras robotisés en passant par les diverses supports de bras mécaniques et électriques) qui soient adaptés à leurs besoins et à un coût juste et maîtrisé, pour pouvoir être accessibles à tous.

- L'importance de promouvoir nos besoins en aides techniques auprès des industriels pour aller plus loin dans l'innovation/le développement de dispositifs d'assistance qui évoluent avec l'évolution de nos pathologies (= aides techniques adaptées aux difficultés rencontrées par les malades).

- L'importance de développer en France des études cliniques sur le sujet en complémentarité avec celles déjà menées en Europe et ailleurs dans le monde.

- La volonté de développer un cercle vertueux: information, prescription, financement et, si refus des organismes payeurs, recours nécessaire, afin que la mise à disposition des outils de compensation de la perte de fonction du membre supérieur deviennent aussi systématique que celle des fauteuils roulants électriques pour la compensation de la perte de la marche.

\section{Conclusion}

Ce colloque qui a permis de réunir tous les acteurs impliqués dans la démarche d'acquisition d'une aide technique de compensation, est aujourd'hui suivi d'un plan d'action, mobilisant toutes les compétences: industriels, distributeurs, prescripteurs, financeurs, associations de patients et les personnes concernées elles-mêmes.

Comme le soulignait Laurence Tiennot-Herment, Présidente de l'AFM-Téléthon lors de son discours de bienvenue: "je suis sûre que ce colloque nous permettra de conclure sur une feuille de route nous permettant, avec des partenaires associatifs confrontés aux mêmes besoins, de sensibiliser et de faire agir dans une même dynamique tous les acteurs concernés par ce sujet hautement prioritaire pour nos malades et nos familles".

L'enjeu majeur de cette feuille de route sera de permettre à chaque malade neuromusculaire, et plus largement à tout malade concerné par une maladie chronique évolutive invalidante, de disposer d'une solution de compensation des membres supérieurs adaptée à ses besoins, sans reste à charge.

Upper limb function loss: the importance of meeting patients with neuromuscular disease's needs

\section{LIENS D'INTÉRÊT}

L'auteur déclare n'avoir aucun lien d'intérêt concernant les données publiées dans cet article.

\section{REMERCIEMENTS}

- À toutes les équipes AFM des différentes directions et Dr J. Andoni Urtizberea pour avoir contribué à l'organisation et à la préparation du colloque.

- Aux différents intervenants : Professeur Samer Al Fayad, Dr Alberto Antonietti, Dr Véronique Bombart, Professeur François Boyer, Mme Corinne Collignon, Dr Charles Fattal, Violaine Guy, Dr Mariska Janssen, Dr Guy Letellier, Samuel Pouplin. - A Aurélie Martin, Benjamin Cornu, Sophie Tricoire et Capucine, sa petite fille.

\section{RÉFÉRENCES}

1. Mise à jour de 21 fiches "techniques produits" distribuées le jour de l'événement à l'ensemble des participants. Disponible en version numérique via le lien suivant : https://www.afm-telethon. $\mathrm{fr} /$ aides-techniques-compensation-membre-superieur-101326 2. Le parcours d'acquisition d'une aide technique : l'expertise des professionnels de santé (film diffusé lors de ce colloque) https://www.afm-telethon.fr/actualites/compensation-membressuperieurs-urgence-priorite-pour-malades-105959

3. Le Jaco de Benjamin (film diffusé lors de ce colloque) https://www.afm-telethon.fr/actualites/compensation-membressuperieurs-urgence-priorite-pour-malades-105959

\section{Retrouvez toutes les Actualités de la Myologie sur les sites de :}

\section{la Société Française de Myologie www.sfmyologie.org}

la filière de santé neuromusculaire FILNEMUS www.filnemus.fr

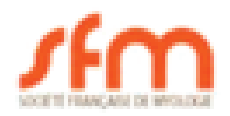

